Method: The data of 32 infants were analysed between October 2014 and March 2015. Further data will be collected between October 2015 and March 2016. Data will then be compared.

Results: Initial data demonstrates the average time infants spent on HFNT was 2.6 days compared to five days in previous studies (Bressan et al 2013).

Discussion: A nurse-led weaning plan has been developed from data collected.

Conclusion: The audit demonstrates a clinical need for efficient weaning of infants on HFNT.

\title{
OC05 - Tokenism or true partnership - parental involvement in a child's acute pain care
}

Jackie Vasey (United Kingdom)ㄹ; Joanna Smith (United Kingdom); Marilynne Kirshbaum (Australia) ${ }^{3}$; Kathleen Chirema (United Kingdom $)^{1}$

${ }^{1}$ University of Huddersfield; ${ }^{2}$ University of Leeds; ${ }^{3}$ Charles Darwin University

Theme: Parenting/parenthood.

Keywords: Acute, child, involvement, pain, parent.

Introduction: Family-centred care (FCC) is widely acknowledged as underpinning children's nursing. Delivering FCC requires nurses to advocate for family involvement, particularly in areas where evidence suggest the child's needs are not being met, such as pain care.

Aim: To explore parental involvement in their child's acute pain care.

Methods: A qualitative ethnographical approach was adopted, with non-participant observation and follow-up interviews. The framework approach underpinned data analysis.

Results: Partnership between nurses and parents is not overtly evident in relation to pain care. Involvement is often 'unspoken', indicating that neither the nurse nor parent are clear about the extent to which parents can, or are, involved in care.

Discussion: Parents want greater involvement in their child's pain care and often initiate involvement by advocating for their child, with or without support from nurses.

Conclusions: The 'Pillars of Partnership in Pain Care' model offers an alternative approach to involving parents.

\section{OC06 - Involving parents in their child's care - where next?}

Joanna Smith (United Kingdom)i; Veronica Swallow (United

Kingdom $)^{1}$; Imelda Coyne (Ireland) ${ }^{2}$

${ }^{1}$ University of Leeds; ${ }^{2}$ Trinity College Dublin

Theme: Complex health care and chronic disease management. 